\title{
IMA Commission on New Minerals, Nomenclature and Classification (CNMNC) NEWSLETTER 49
}

\section{New minerals and nomenclature modifications approved in 2019}

\author{
Ritsuro Miyawaki (Chairman, CNMNC) ${ }^{1}$, Frédéric Hatert (Vice-Chairman, CNMNC)2 , Marco Pasero (Vice-Chairman, \\ CNMNC) ${ }^{3 *}$ and Stuart J. Mills (Secretary, CNMNC) ${ }^{4}$ \\ ${ }^{1}$ Department of Geology and Paleontology, National Museum of Nature and Science, 4-1-1 Amakubo, Tsukuba 305-0005, Japan - miyawaki@kahaku.go.jp; \\ ${ }^{2}$ Laboratoire de Minéralogie, Université de Liège, B-4000 Liège, Belgium - fhatert@uliege.be; ${ }^{3}$ Dipartimento di Scienze della Terra, Università di Pisa, Via Santa Maria \\ 53, I-56126 Pisa, Italy - marco.pasero@unipi.it; and ${ }^{4}$ Geosciences, Museums Victoria, PO Box 666, Melbourne, Victoria 3001, Australia - smills@museum.vic.gov.au
}

The information given here is provided by the IMA Commission on New Minerals, Nomenclature and Classification for comparative purposes and as a service to mineralogists working on new species.

Each mineral is described in the following format:

Mineral name, if the authors agree on its release prior to the full description appearing in press

Chemical formula

Type locality

Full authorship of proposal

E-mail address of corresponding author

Relationship to other minerals

Crystal system, Space group; Structure determined, yes or no

Unit-cell parameters

Strongest lines in the powder X-ray diffraction pattern

Type specimen repository and specimen number

Citation details for the mineral prior to publication of full description

Citation details concern the fact that this information will be published in the Mineralogical Magazine on a routine basis, as well as being added month by month to the Commission's web site.

It is still a requirement for the authors to publish a full description of the new mineral.

\section{NO OTHER INFORMATION WILL BE RELEASED BY THE COMMISSION}

\section{NEW MINERAL PROPOSALS APPROVED IN APRIL 2019}

IMA No. 2018-141

Andymcdonaldite

$\mathrm{Fe}_{2} \mathrm{TeO}_{6}$

Wildcat prospect, NW portion of the Detroit District, Juab Co., Utah, USA

Mati Raudsepp* ${ }^{\star}$ Mark F. Coolbaugh, John K. McCormack, Edith Czech and Rhy McMillan

*E-mail: mraudsepp@eoas.ubc.ca

Known synthetic analogue

Tetragonal: $P 4_{2} / \mathrm{mnm}$

$a=4.6222(9), c=9.077(3) \AA$

4.538(12), 4.119(35), 3.268(100), 2.652(10), 2.531(58), 2.311(13),

$1.707(48), 1.634(14)$

Type material is deposited in the mineralogical collections of the Smithsonian National Museum of Natural History, 10th St. \& Constitution Ave., Washington, DC 20560, USA, catalogue number NMNH 177134

\footnotetext{
${ }^{*}$ Author for correspondence: Marco Pasero, Email: marco.pasero@unipi.it

Cite this article: Miyawaki R., Hatert F., Pasero M. and Mills S.J. (2019) New minerals and nomenclature modifications approved in 2019. Mineralogical Magazine 83, 479-483. https://doi.org/10.1180/mgm.2019.35
}

How to cite: Raudsepp, M., Coolbaugh, M.F., McCormack, J.K., Czech, E. and McMillan, R. (2019) Andymcdonaldite, IMA 2018-141. CNMNC Newsletter No. 49: Mineralogical Magazine, 83, doi:10.1180/mgm.2019.35.

IMA No. 2018-142

Aluminosugilite $\mathrm{KNa}_{2} \mathrm{Al}_{2} \mathrm{Li}_{3} \mathrm{Si}_{12} \mathrm{O}_{30}$

Cerchiara mine, Faggiona, Borghetto Vara-Pignone, La Spezia, Liguria, Italy $\left(44^{\circ} 11^{\prime} 57^{\prime \prime} \mathrm{N}, 9^{\circ} 42^{\prime} 1^{\prime \prime} \mathrm{E}\right)$

Mariko Nagashima*, Chihiro Fukuda, Takashi Matsumoto, Teruyoshi Imaoka, Gianluca Odicino and Gianluca Armellino

*E-mail: nagashim@yamaguchi-u.ac.jp

Osumilite group

Hexagonal: $P 6 / m c c$; structure determined

$a=9.9830(4), c=13.9667(5) \AA$

4.32(100), 4.06(38), 3.67(14), 3.48(30), 3.18(92), 2.86(70), 2.71(18), 2.49(18)

Type material is deposited in the mineralogical collections of the National Museum of Nature and Science, Tsukuba, Japan, specimen numbers NSM-MF-16503 
How to cite: Nagashima, M., Fukuda, C., Matsumoto, T., Imaoka, T., Odicino, G. and Armellino, G. (2019) Aluminosugilite, IMA 2018-142. CNMNC Newsletter No. 49: Mineralogical Magazine, 83, doi:10.1180/mgm.2019.35.

IMA No. 2018-150

Siwaqaite

$\mathrm{Ca}_{6} \mathrm{Al}_{2}\left(\mathrm{CrO}_{4}\right)_{3}(\mathrm{OH})_{12} \cdot 26 \mathrm{H}_{2} \mathrm{O}$

North Siwaqa complex, Lisdan-Siwaqa Fault, Hatrurim Complex, ca. $60 \mathrm{~km} \mathrm{~S}$ of Amman, Jordan $\left(31^{\circ} 24^{\prime} 15^{\prime \prime} \mathrm{N}, 36^{\circ}\right.$ 14 '34'E)

Rafał Juroszek*, Biljana Krüger, Irina O. Galuskina, Hannes Krüger, Yevgeny Vapnik and Evgeny V. Galuskin

*E-mail: rjuroszek@us.edu.pl

The $\mathrm{Cr}$ analogue of ettringite

Trigonal: $P 31 c$; structure determined

$a=11.3640(2), c=21.4485(2) \AA$

$9.841(100), \quad 5.682(65), \quad 5.021(16), \quad 4.709(38), \quad 3.900(29)$, $3.280(17), 3.279(33), 2.224(16)$

Type material is deposited in the collections of the Fersman Mineralogical Museum, Russian Academy of Sciences, Leninskiy Prospekt 18-2, Moscow 119071, Russia, registration number 5277/1

How to cite: Juroszek, R., Krüger, B., Galuskina, I.O., Krüger, H., Vapnik, Y. and Galuskin, E.V. (2019) Siwaqaite, IMA 2018-150. CNMNC Newsletter No. 49: Mineralogical Magazine, 83, doi:10.1180/mgm.2019.35.

\section{IMA No. 2018-154}

Luxembourgite

$\mathrm{AgCuPbBi}_{4} \mathrm{Se}_{8}$

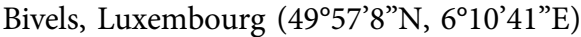

Simon Philippo, Frédéric Hatert*, Yannick Bruni and Pietro Vignola

*E-mail: fhatert@uliege.be

Isostructural with watkinsonite and litochlebite

Monoclinic: $P 2_{1} / m$; structure determined

$a=13.002(1), b=4.1543(3), c=15.312(2) \AA, \beta=108.92(1)^{\circ}$

4.61(20), 3.59(20), 2.984(100), 2.425(20), 2.085(60), 1.916

(20), 1.355(30), 1.188(30)

Type material is deposited in the mineralogical collections of the Natural History Museum of Luxembourg, Rue Münster 25, L-2560 Luxembourg, Luxembourg, catalogue number FD040, and the Laboratory of Mineralogy, University of Liège, Bâtiment B18, Sart Tilman, B-4000 Liège, Belgium, catalogue number 21302

How to cite: Philippo, S., Hatert, F., Bruni, Y. and Vignola, P. (2019) Luxembourgite, IMA 2018-154. CNMNC Newsletter No. 49: Mineralogical Magazine, 83, doi:10.1180/mgm.2019.35.

\section{IMA No. 2018-156}

Tsikourasite

$\mathrm{Mo}_{3} \mathrm{Ni}_{2} \mathrm{P}_{1+x}(x<0.25)$

Agios Stefanos mine, ca. $10 \mathrm{~km} \mathrm{~S}$ of the Domokos village, Othrys ophiolite, Greece (394'59"N, 22²5'59”E)

Federica Zaccarini* ${ }^{*}$, Luca Bindi, Elena Ifandi, Tassos Grammatikopoulos, Chris J. Stanley, Giorgio Garuti and Daniela Mauro
^E-mail: Federica.Zaccarini@unileoben.ac.at

Known synthetic analogue

Cubic: $F \overline{4} 3 m$; structure determined

$a=10.8215(5) \AA$

$2.705(20), \quad 2.483(18), 2.209(65), 2.083(100), \quad 1.913(32)$,

1.803(12), 1.275(26), 1.089(12)

Type material is deposited in the mineralogical collections of the Museo di Storia Naturale, Università di Firenze, Via La Pira 4, I-50121, Firenze, Italy, catalogue number 3296/I How to cite: Zaccarini, F., Bindi, L., Ifandi, E. Grammatikopoulos, T., Stanley, C.J., Garuti, G. and Mauro, D. (2019) Tsikourasite, IMA 2018-156. CNMNC Newsletter No. 49: Mineralogical Magazine, 83, doi:10.1180/mgm.2019.35.

\section{IMA No. 2018-157}

Laurentthomasite

$\mathrm{Mg}_{2} \mathrm{~K}\left(\mathrm{Be}_{2} \mathrm{Al}\right) \mathrm{Si}_{12} \mathrm{O}_{30}$

$40 \mathrm{~km} \mathrm{E}$ of the village of Betroka, in a rural area named Beravina, close to the small village of Ambaro, Toliara province, Madagascar (23⒉ $\left.1^{\prime} 0^{\prime} S, 46^{\circ} 25^{\prime} 0^{\prime \prime} \mathrm{E}\right)$

Cristiano Ferraris*, Isabella Pignatelli, Fernando Cámara, Sylvain Ponti, Martin Schreyer, Gian Carlo Parodi and Fengxia Wei

*E-mail: ferraris@mnhn.fr

The $\mathrm{Mg}$ analogue of milarite

Hexagonal: $P 6 / m c c$; structure determined

$a=9.95343(6), c=14.15583(8) \AA$

7.055(2), 4.965(6), 4.302(2), 4.064(8), 3.533(3), 3.171(10),

2.881(8), 2.372(4)

Type material is deposited in the mineralogical collections of the Muséum National d'Histoire Naturelle, 61 rue Buffon, 75005 Paris, France, catalogue numbers 218.1_a (holotype), 218.1_b and 218.1_c (cotypes)

How to cite: Ferraris, C., Pignatelli, I., Cámara, F., Ponti, S., Schreyer, M., Parodi, G.C. and Wei, F. (2019) Laurentthomasite, IMA 2018-157. CNMNC Newsletter No. 49: Mineralogical Magazine, 83, doi:10.1180/mgm.2019.35.

IMA No. 2018-158

Kahlenbergite

$\mathrm{KAl}_{11} \mathrm{O}_{17}$

Ca. $300 \mathrm{~m}$ NE of the Parsa Mt, Negev Desert, Hatrurim Basin, Israel $\left(31^{\circ} 12.18^{\prime} \mathrm{N}, 36^{\circ} 15.31^{\prime} \mathrm{E}\right)$

Biljana Krüger ${ }^{\star}$, Evgeny V. Galuskin, Irina O. Galuskina, Hannes Krüger and Yevgeny Vapnik

${ }^{\star}$ E-mail: biljana.krueger@uibk.ac.at

The $\mathrm{K}$ analogue of diaoyudaoite

Hexagonal: $P 6_{3} / m m c$; structure determined

$a=5.64860(6), c=22.8970(3) \AA$

$11.448(100), \quad 5.724(15), 2.824(14), 2.719(34), 2.533(26)$, 2.059(12), 1.610(13), 1.412(19)

Type material is deposited in the collections of the Fersman Mineralogical Museum, Russian Academy of Sciences, Leninskiy Prospekt 18-2, Moscow 119071, Russia, registration number 5295/1

How to cite: Krüger, B., Galuskin, E.V., Galuskina, I.O., Krüger, H. and Vapnik, Y. (2019) Kahlenbergite, IMA 2018-158. CNMNC Newsletter No. 49: Mineralogical Magazine, 83, doi:10.1180/mgm.2019.35. 
IMA No. 2018-159

Scorticoite

$\mathrm{Mn}_{6}(\mathrm{Sb} \square)_{\Sigma 2}\left(\mathrm{SiO}_{4}\right)_{2} \mathrm{O}_{3}(\mathrm{OH})_{3}$

Scortico Mn ore deposit, Fivizzano, Apuan Alps, Massa

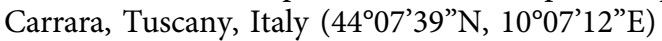

Cristian Biagioni*, Elena Bonaccorsi, Anthony R. Kampf,

Federica Zaccarini, Ulf Hålenius and Ferdinando Bosi

^E-mail: cristian.biagioni@unipi.it

Welinite group

Trigonal: $P 3$; structure determined

$a=8.205(1), c=4.774(1) \AA$

3.105(s), 2.858(s), 2.676(ms), 2.332(ms), 1.787(s), 1.685(m),

$1.552(\mathrm{~m})$

Type material is deposited in the mineralogical collections of the Museo di Storia Naturale, Università di Pisa, Via Roma 79, Calci, Pisa, Italy, catalogue number 19908

How to cite: Biagioni, C., Bonaccorsi, E., Kampf, A.R., Zaccarini, F., Hålenius, U. and Bosi, F. (2019) Scorticoite, IMA 2018-159. CNMNC Newsletter No. 49: Mineralogical Magazine, 83, doi:10.1180/mgm.2019.35.

\section{IMA No. 2018-160}

Potassic-hastingsite

$\mathrm{KCa}_{2}\left(\mathrm{Fe}_{4}^{2+} \mathrm{Fe}^{3+}\right)\left(\mathrm{Si}_{6} \mathrm{Al}_{2}\right) \mathrm{O}_{22}(\mathrm{OH})_{2}$

Danailingou, Tongxing town, Keshiketeng Banner, Inner

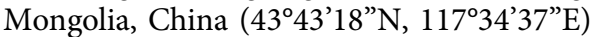

Guangming Ren*, Guowu Li, Jiaxin Shi, Xiangping Gu, Guang Fan, Apeng Yu, Qiuxiao Liu and Ganfu Shen

^E-mail: rguangming928@sina.com

Amphibole supergroup

Monoclinic: $C 2 / \mathrm{m}$; structure determined

$a=9.9405(7), b=18.256(2), c=5.3501(3) \AA, \beta=105.117(5)^{\circ}$

$8.504(100), 3.417(36), 3.155(63), 2.735(71), 2.623(35)$,

2.570(34), 2.365(29), 2.179(30)

Type material is deposited in the mineralogical collections of the Geological Museum of China, Beijing, China, catalogue number M13815

How to cite: Ren, G., Li, G., Shi, J., Gu, X., Fan, G., Yu, A., Liu, Q. and Shen, G. (2019) Potassic-hastingsite, IMA 2018-160. CNMNC Newsletter No. 49: Mineralogical Magazine, 83, doi:10.1180/mgm.2019.35.

\section{IMA No. 2018-161}

Rumoiite

$\mathrm{AuSn}_{2}$

Minamichiyoda, Shosanbetsu village, Rumoi Subprefecture,

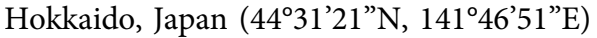

Daisuke Nishio-Hamane* and Katsuyuki Saito

${ }^{\star}$ E-mail: hamane@issp.u-tokyo.ac.jp

Known synthetic analogue

Orthorhombic: $\mathrm{Pbca}$

$a=6.905(2), b=7.017(2), c=11.797(3) \AA$

$4.542(58), \quad 3.774(48), \quad 2.950(100), \quad 2.711(52), \quad 2.409(41)$, 2.244(56), 2.136(47), 2.123(65)

Type material is deposited in the mineralogical collections of the National Museum of Nature and Science, Tsukuba, Japan, specimen number NSM-M46178 (holotype) and M46179 (cotype) How to cite: Nishio-Hamane, D. and Saito, K. (2019) Rumoiite, IMA 2018-161. CNMNC Newsletter No. 49: Mineralogical Magazine, 83, doi:10.1180/mgm.2019.35.
IMA No. 2018-162

Shosanbetsuite

$\mathrm{Ag}_{3} \mathrm{Sn}$

Minamichiyoda, Shosanbetsu village, Rumoi Subprefecture,

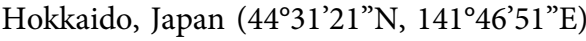

Daisuke Nishio-Hamane ${ }^{\star}$ and Katsuyuki Saito

^E-mail: hamane@issp.u-tokyo.ac.jp

Known synthetic analogue

Orthorhombic: Pmmn

$a=5.998(5), b=4.7736(9), c=5.154(3) \AA$

2.592(10), 2.586(7), 2.389(30), 2.275(100), 2.267(85), 1.756(78), $1.356(73), 1.354(53)$

Type material is deposited in the mineralogical collections of the National Museum of Nature and Science, Tsukuba, Japan, specimen number NSM-M46178 (holotype) and M46179 (cotype)

How to cite: Nishio-Hamane, D. and Saito, K. (2019) Shosanbetsuite, IMA 2018-162. CNMNC Newsletter No. 49: Mineralogical Magazine, 83, doi:10.1180/mgm.2019.35.

IMA No. 2018-165

Giacovazzoite

$\mathrm{K}_{5} \mathrm{Fe}_{3}^{3+} \mathrm{O}\left(\mathrm{SO}_{4}\right)_{6} \cdot 10 \mathrm{H}_{2} \mathrm{O}$

Monte Arsiccio mine, Stazzema (LU), Apuan Alps, Tuscany, Italy $\left(43^{\circ} 58^{\prime} \mathrm{N}, 10^{\circ} 17^{\prime} \mathrm{E}\right)$

Cristian Biagioni ${ }^{\star}$, Luca Bindi, Daniela Mauro and Marco Pasero

*E-mail: cristian.biagioni@unipi.it

Known synthetic analogue

Monoclinic: $P 2_{1} / c$; structure determined

$a=9.4797(2), b=18.4454(5), c=18.0540(4) \AA, \beta=92.626(2)^{\circ}$

9.1(s), 8.2(vs), 4.15(mw), 4.02(mw), 3.442(m), 3.371(m), 3.005(m), 2.968(m)

Type material is deposited in the mineralogical collections of the Museo di Storia Naturale, Università di Pisa, Via Roma 79, Calci, Pisa, Italy, catalogue number 19896

How to cite: Biagioni, B., Bindi, L., Mauro, D. and Pasero, M. (2019) Giacovazzoite, IMA 2018-165. CNMNC Newsletter No. 49: Mineralogical Magazine, 83, doi:10.1180/mgm.2019.35.

\section{IMA No. 2018-166}

Pseudomeisserite- $\left(\mathrm{NH}_{4}\right)$

$\left(\mathrm{NH}_{4}\right)_{2} \mathrm{Na}_{4}\left[\left(\mathrm{UO}_{2}\right)_{2}\left(\mathrm{SO}_{4}\right)_{5}\right] \cdot 4 \mathrm{H}_{2} \mathrm{O}$

Blue Lizard mine, Red Canyon, White Canyon District, San

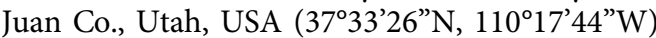

Anthony R. Kampf*, Travis A. Olds, Jakub Plášil, Barbara P. Nash and Joe Marty

^E-mail: akampf@nhm.org

New structure type

Monoclinic: $P 2_{1} / c$; structure determined

$a=13.1010(3), b=10.0948(2), c=19.494(1) \AA, \beta=104.285(7)^{\circ}$ 12.69(76), 8.88(55), 6.83(84), 6.01(100), 4.593(51), 4.414(57), $3.959(67), 3.135(76)$

Cotype material is deposited in the mineralogical collections of the Natural History Museum of Los Angeles County, 900 Exposition Boulevard, Los Angeles, CA 90007, USA, catalogue numbers 67621 and 67622 
How to cite: Kampf, A.R., Olds, T.A., Plášil, J., Nash, B.P. and Marty, J. (2019) Pseudomeisserite- $\left(\mathrm{NH}_{4}\right)$, IMA 2018-166. CNMNC Newsletter No. 49: Mineralogical Magazine, 83, doi:10.1180/mgm.2019.35.

IMA No. 2018-167

Gobelinite

$\mathrm{CoCu}_{4}\left(\mathrm{SO}_{4}\right)_{2}(\mathrm{OH})_{6} \cdot 6 \mathrm{H}_{2} \mathrm{O}$

Cap Garonne mine (pillar $78 \mathrm{~b}$ in the north mine), Var, Provence-Alpes-Côte d'Azur, France ( $43^{\circ} 4^{\prime} 53^{\prime \prime} \mathrm{N}, 6^{\circ} 1^{\prime} 55^{\prime \prime} \mathrm{E}$ - type locality); Schlänger und Eichert vein of the Eisenzecher Zug mine, Eiserfeld, Siegerland, North Rhine-Westphalia, Germany (5049'9”N, 759'14"E - cotype locality)

Stuart J. Mills*, Uwe Kolitsch, Georges Favreau, William D. Birch, Valérie Galea-Clolus and Johannes Markus Henrich

*E-mail: smills@museum.vic.gov.au

The Co analogue of ktenasite

Monoclinic: $P 2_{1} / c$; structure determined

$a=5.599(1), b=6.084(1), c=23.676(5) \AA, \beta=95.22(3)^{\circ}$

$11.870(100), \quad 5.924(40), 4.883(10), \quad 4.825(15), \quad 3.946(15)$, 2.956(15), 2.663(20), 2.561(15)

Type material is deposited in the mineralogical collections of Geosciences, Museums Victoria, GPO Box 666, Melbourne, Victoria 3001, Australia, registration number M54565 (Cap Garonne), and the Naturhistorisches Museum, Burgring 7, A-1010 Wien, Austria, registration number O 1045 (Eisenzecher Zug)

How to cite: Mills, S.J., Kolitsch, U., Favreau, G., Birch, W.D., Galea-Clolus, V. and Henrich, J.M. (2019) Gobelinite, IMA 2018-167. CNMNC Newsletter No. 49: Mineralogical Magazine, 83, doi:10.1180/mgm.2019.35.

\section{IMA No. 2018-108a}

Fluorapophyllite-(Cs)

$\mathrm{CsCa}_{4}\left(\mathrm{Si}_{8} \mathrm{O}_{20}\right) \mathrm{F}\left(\mathrm{H}_{2} \mathrm{O}\right)_{8}$

Darai-Pioz glacier, Alai mountain range, Tien-Shan, Rashtskii district, Tajikistan $\left(39^{\circ} 30^{\prime} \mathrm{N}, 70^{\circ} 40^{\prime} \mathrm{E}\right)$

Atali A. Agakhanov, Leonid A. Pautov, Anatoly V. Kasatkin, Elena Sokolova, Maxwell C. Day, Frank C. Hawthorne, Vladimir Y. Karpenko, Vyacheslav A. Muftakhov, Igor V. Pekov, Fernando Cámara Artigas and Sergey N. Britvin *E-mail: atali99@mail.ru

Apophyllite group

Tetragonal: $P 4 / \mathrm{nmc}$

$a=9.060(6), c=15.741(11) \AA$

$7.870(100), 3.935(100), 3.602(55), 2.974(84), 2.515(73)$, 2.486(71), 2.119(42), 2.030(45)

Type material is deposited in the collections of the Fersman Mineralogical Museum, Russian Academy of Sciences, Leninskiy Prospekt 18-2, Moscow 119071, Russia, registration number 5280/1

How to cite: Agakhanov, A.A., Pautov, L.A., Kasatkin, A.V., Sokolova, E., Day, M.C., Hawthorne, F.C., Karpenko, V.Y., Muftakhov, V.A., Pekov, I.V., Cámara Artigas, F. and Britvin, S.N. (2019) Fluorapophyllite-(Cs), IMA 2018-108a. CNMNC Newsletter No. 49: Mineralogical Magazine, 83, doi:10.1180/mgm.2019.35.

\section{NEW MINERAL PROPOSALS APPROVED IN MAY 2019}

IMA No. 2019-001

Smamite

$\mathrm{Ca}_{2} \mathrm{Sb}(\mathrm{OH})_{4}\left[\mathrm{H}\left(\mathrm{AsO}_{4}\right)_{2}\right] \cdot 6 \mathrm{H}_{2} \mathrm{O}$

Giftgrube mine, St. Jacques vein, Rauenthal, Sainte Marie-aux-Mines, Haut-Rhin province, France $\left(48^{\circ}\right.$ $\left.13^{\prime} 28^{\prime} \mathrm{N}, 7^{\circ} 9^{\prime} 7^{\prime \prime} \mathrm{E}\right)$

Jakub Plášil ${ }^{\star}$, Anthony R. Kampf, Nicolas Meisser, Cédric Lheur, Thierry Brunsperger and Radek Škoda

*E-mail: plasil@fzu.cz

New structure type

Triclinic: $P \overline{1}$; structure determined

$a=6.8207(4), b=8.0959(4), c=8.2130(6) \AA, \alpha=95.834(7)$,

$\beta=110.762(8), \gamma=104.012(7)^{\circ}$

7.56(41), 6.03(60), 5.66(47), 5.07(100), 3.992(43), 3.783(36), 2.858(51), 2.766(31)

Type material is deposited in the mineralogical collections of the Musée Cantonal de Géologie, University of Lausanne, Anthropole, Dorigny, CH-1015 Lausanne, Switzerland, catalogue number MGL 093481 (holotype), 093482 and 093481 (cotypes), and the Natural History Museum of Los Angeles County, 900 Exposition Boulevard, Los Angeles, CA 90007, USA, catalogue number 67169 (cotype)

How to cite: Plášil, J., Kampf, A.R., Meisser, N., Lheur, C., Brunsperger, T. and Škoda, R. (2019) Smamite, IMA 2019-001. CNMNC Newsletter No. 49: Mineralogical Magazine, 83, doi:10.1180/mgm.2019.35.

IMA No. 2019-002

Caseyite

$\left[\left(\mathrm{V}^{5+} \mathrm{O}_{2}\right) \mathrm{Al}_{7.5}(\mathrm{OH})_{15}\left(\mathrm{H}_{2} \mathrm{O}\right)_{13}\right]_{2}\left[\mathrm{H}_{2} \mathrm{~V}^{4+} \mathrm{V}_{9}^{5+} \mathrm{O}_{28}\right]\left[\mathrm{V}_{10}^{5+} \mathrm{O}_{28}\right]_{2} \cdot 90 \mathrm{H}_{2} \mathrm{O}$ West Sunday mine, Slick Rock district, San Miguel Co., Colorado, USA $\left(38^{\circ} 4^{\prime} 48.03^{\prime} \mathrm{N}, 108^{\circ} 49^{\prime} 18.07^{\prime \prime} \mathrm{W}\right)$; Packrat mine, near Gateway, Mesa Co., Colorado, USA $\left(38^{\circ}\right.$ 38'51.28”N, $\left.109^{\circ} 2^{\prime} 49.77^{\prime \prime} W\right)$; Burro mine, Slick Rock district, San Miguel Co., Colorado, USA (38²'42”N, 108 53'23”W)

Anthony R. Kampf*, Mark A. Cooper, John M. Hughes, Barbara P. Nash, Joe Marty and Frank C. Hawthorne

*E-mail: akampf@nhm.org

New structure type

Monoclinic: $P 2{ }_{1} / n$; structure determined $a=14.123(8), b=31.00(1), c=21.95(1) \AA, \beta=97.961(8)^{\circ}$ 17.798(92), 15.499(100), 12.749(26), 12.620(33), 10.869(16), 9.332(11), 9.016(14), 8.899(43)

Type material is deposited in the mineralogical collections of the Natural History Museum of Los Angeles County, 900 Exposition Boulevard, Los Angeles, CA 90007, USA, catalogue numbers 73526 (Packrat - holotype), 73527 and 73528 (Packrat - cotypes), 73529 (West Sunday - cotype), 73530 and 73531 (Burro - cotypes)

How to cite: Kampf, A.R., Cooper, M.A., Hughes, J.M., Nash, B.P., Marty, J. and Hawthorne, F.C. (2019) Caseyite, IMA 2019-002. CNMNC Newsletter No. 49: Mineralogical Magazine, 83, doi:10.1180/mgm.2019.35.

\section{IMA No. 2019-005}

Langhofite

$\mathrm{Pb}_{2}(\mathrm{OH})\left[\mathrm{WO}_{4}(\mathrm{OH})\right]$

Långban deposit, Filipstad district, Värmland, Sweden $\left(59.85^{\circ} \mathrm{N}, 14.26^{\circ} \mathrm{E}, 215 \mathrm{~m}\right.$ a.s.l.) 
Dan Holtstam, Fernando Cámara and Andreas Karlsson *E-mail: dan.holtstam@nrm.se

New structure type

Triclinic: $P \overline{1}$; structure determined

$a=6.6118(3), b=7.0748(4), c=7.3264(4) \AA, \alpha=118.125(6)$,

$\beta=94.503(5), \gamma=101.146(5)^{\circ}$

$6.04(24), \quad 3.330(17), \quad 3.259(22), \quad 3.181(19), \quad 3.079(24)$,

3.016(100), 2.053(20), 2.050(18)

Type material is deposited in the mineralogical collections of the Department of Geosciences, Swedish Museum of Natural History, Box 50007, SE-104 05 Stockholm, Sweden, collection number GEO-NRM 20030044

How to cite: Holtstam, D., Cámara, F. and Karlsson, A. (2019) Langhofite, IMA 2019-005. CNMNC Newsletter No. 49: Mineralogical Magazine, 83, doi:10.1180/mgm.2019.35.

IMA No. 2019-006

Magnanelliite

$\mathrm{K}_{3} \mathrm{Fe}_{2}^{3+}\left(\mathrm{SO}_{4}\right)_{4}(\mathrm{OH})\left(\mathrm{H}_{2} \mathrm{O}\right)_{2}$

Monte Arsiccio mine, Stazzema, Apuan Alps, Tuscany, Italy $\left(43^{\circ} 58^{\prime} \mathrm{N}, 10^{\circ} 17^{\prime} \mathrm{E}\right)$

Cristian Biagioni, Luca Bindi and Anthony R. Kampf

*E-mail: cristian.biagioni@unipi.it

The $\mathrm{Fe}^{3+}$ analogue of alcaparrosaite

Monoclinic: $C 2 / c$; structure determined

$a=7.5491(3), b=16.8652(6), c=12.1574(4) \AA, \beta=94.064$

$(1)^{\circ}$

6.9(m), 4.91(mw), 3.612(mw), 3.427(mw), 3.300(mw), 3.085

(s), 3.006(m), 2.704(m)

Type material is deposited in the mineralogical collections of the Museo di Storia Naturale, Università di Pisa, Via Roma 79, Calci (Pisa, Italy), catalogue number 19894 (holotype), and the Natural History Museum of Los Angeles County, 900 Exposition Boulevard, Los Angeles, CA 90007, USA, catalogue number 67241 (cotype)

How to cite: Biagioni, C., Bindi, L. and Kampf, A.R. (2019) Magnanelliite, IMA 2019-006. CNMNC Newsletter No. 49: Mineralogical Magazine, 83, doi:10.1180/mgm.2019.35.

\section{NOMENCLATURE PROPOSALS APPROVED IN MARCH 2019}

IMA 18-C: Redefinition of calumetite and discreditation of vondechenite

Proposal 18-C is accepted, and calumetite is redefined from $\mathrm{Cu}(\mathrm{OH}, \mathrm{Cl})_{2} \cdot 2 \mathrm{H}_{2} \mathrm{O}$ to $\mathrm{CaCu}_{4}(\mathrm{OH})_{8} \mathrm{Cl}_{2} \cdot 3 \cdot 5 \mathrm{H}_{2} \mathrm{O}$. Vondechenite (IMA2016-65), identical to calumetite, is consequently discredited.

IMA 18-I: Nomenclature of minerals of the rhodonite group Proposal 18-I is accepted, and the rhodonite group is established. Currently it consists of two mineral species having the following end-member formulae: rhodonite, ${ }^{\mathrm{M} 5} \mathrm{Ca}^{\mathrm{M} 1-\mathrm{M} 3} \mathrm{Mn}_{3}{ }^{\mathrm{M} 4} \mathrm{MnSi}_{5} \mathrm{O}_{15}$, and ferrorhodonite ${ }^{\mathrm{M} 5} \mathrm{Ca}^{\mathrm{M} 1-\mathrm{M} 3} \mathrm{Mn}_{3}{ }^{\mathrm{M} 4} \mathrm{FeSi}_{5} \mathrm{O}_{15}$.

IMA 19-A: Redefinition of tsugaruite

Proposal 19-A is accepted, and tsugaruite is redefined as a lead-arsenic chloro-sulfosalt, having ideal formula $\mathrm{Pb}_{28} \mathrm{As}_{15} \mathrm{~S}_{50} \mathrm{Cl}$.

\section{NOMENCLATURE PROPOSALS APPROVED IN APRIL 2019}

IMA 18-H: Redefinition of cadwaladerite and discreditation of lesukite

Proposal $18-\mathrm{H}$ is accepted, demonstrating that cadwaladerite and lesukite are the same mineral species. Because cadwaladerite has historical precedence, it is recognised as a valid mineral species, whereas lesukite is discredited. Furthermore, the formula of cadwaladerite is redefined as $\mathrm{Al}_{5}\left(\mathrm{H}_{2} \mathrm{O}\right)_{3}(\mathrm{OH})_{12} \cdot n\left(\mathrm{Cl}, \mathrm{H}_{2} \mathrm{O}\right)$.

IMA 18-K: Nomenclature and classification of the tetrahedrite group

Proposal $18-\mathrm{K}$ on the tetrahedrite group is accepted. The general structural formula of minerals belonging to this group is ${ }^{M(2)} \mathrm{A}_{6}{ }^{M(1)}\left(\mathrm{B}_{4} \mathrm{C}_{2}\right)_{\Sigma 6}{ }^{X(3)} \mathrm{D}_{4}{ }^{\mathrm{S}(1)} \mathrm{Y}_{12}{ }^{\mathrm{S}(2)} \mathrm{Z}(Z=2)$, where $\mathrm{A}=$ $\mathrm{Cu}^{+}, \mathrm{Ag}^{+}, \square ; \mathrm{B}=\mathrm{Cu}^{+}, \mathrm{Ag}^{+} ; \mathrm{C}=\mathrm{Zn}^{2+}, \mathrm{Fe}^{2+}, \mathrm{Hg}^{2+}, \mathrm{Cd}^{2+}, \mathrm{Mn}^{2}$ ${ }^{+}, \mathrm{Cu}^{2+}, \mathrm{Cu}^{+}, \mathrm{Fe}^{3+} ; \mathrm{D}=\mathrm{Sb}^{3+}, \mathrm{As}^{3+}, \mathrm{Bi}^{3+}, \mathrm{Te}^{4+} ; \mathrm{Y}=\mathrm{S}^{2-}, \mathrm{Se}^{2-}$; and $\mathrm{Z}=\mathrm{S}^{2-}, \mathrm{Se}^{2-}, \square$. The tetrahedrite group is divided into five different series on the basis of the $\mathrm{A}, \mathrm{B}, \mathrm{D}$, and $\mathrm{Y}$ constituents, i.e. the tetrahedrite, tennantite, freibergite, hakite, and giraudite series. The nature of the dominant $\mathrm{C}$ constituent is made explicit using a hyphenated suffix between parentheses. Eleven minerals belonging to the tetrahedrite group can be considered as valid species: argentotennantite- $(\mathrm{Zn})$, argentotetrahedrite- $(\mathrm{Fe}), \quad$ kenoargentotetrahedrite- $(\mathrm{Fe})$, giraudite- $(\mathrm{Zn})$, goldfieldite, hakite- $(\mathrm{Hg})$, rozhdestvenskayaite- $(\mathrm{Zn}), \quad$ tennantite- $(\mathrm{Fe}), \quad$ tennantite- $(\mathrm{Zn})$, tetrahedrite-(Fe), and tetrahedrite-(Zn). Furthermore, annivite is formally discredited.

IMA 19-B: Establishment of the calcioferrite group

Proposal 19-B on the calcioferrite group is accepted. The general formula of minerals belonging to this group is $\mathrm{Ca}_{4} A B_{4}\left(\mathrm{PO}_{4}\right)_{6}(\mathrm{OH})_{4} \cdot 12 \mathrm{H}_{2} \mathrm{O}$, and two subgroups are defined on the basis of the dominant cation at the $B$ site: the calcioferrite subgroup $\left(B=\mathrm{Fe}^{3+}\right.$ : calcioferrite, zodacite), and the montgomeryite subgroup ( $B=\mathrm{Al}$ : montgomeryite, kingsmountite, fanfaniite). Aniyunwiyaite is discredited as being kingsmountite, which is assigned a triclinic structure with an ideal formula changed to $\mathrm{Ca}_{3} \mathrm{MnFeAl}_{4}\left(\mathrm{PO}_{4}\right)_{6}(\mathrm{OH})_{4} \cdot 12 \mathrm{H}_{2} \mathrm{O}$.

\section{NOMENCLATURE PROPOSALS APPROVED IN MAY 2019}

Application of the IMA-CNMNC dominant-valency rule to complex mineral compositions

This is a special proposal, coauthored by all the officers of the CNMNC and approved by the members of the Commission itself. It is aimed at solving possible troubles in the definition of the ideal end-member formula of a mineral, which may be crucial for the definition of new mineral species. The IMA-CNMNC recommends the use of the dominant-valency rule for mineral nomenclature, because it alone may lead to unambiguous mineral identification. However, the simple application of the dominant-valency rule may sometimes result in unbalanced end-member formulae, due to the occurrence of a coupled heterovalent substitution at two sites along with a heterovalent substitution at a single site. In these cases, it is recommended to use the site total charge approach to identify the dominant root-charge arrangement on which the dominantconstituent rule can be applied. 